\title{
INTEGRAL EQUATION METHODS FOR UNSTEADY STOKES FLOW IN TWO DIMENSIONS
}

\author{
SHIDONG JIANG *, SHRAVAN VEERAPANENI ${ }^{\dagger}$, AND LESLIE GREENGARD $\ddagger$
}

\begin{abstract}
We present an integral equation formulation for the unsteady Stokes equations in two dimensions. This problem is of interest in its own right, as a model for slow viscous flow, but perhaps more importantly, as an ingredient in the solution of the full, incompressible Navier-Stokes equations. Using the unsteady Green's function, the velocity evolves analytically as a divergence-free vector field. This avoids the need for either the solution of coupled field equations (as in fully implicit PDE-based marching schemes) or the projection of the velocity field onto a divergence free field at each time step (as in operator splitting methods). In addition to discussing the analytic properties of the operators that arise in the integral formulation, we describe a family of high-order accurate numerical schemes and illustrate their performance with several examples.
\end{abstract}

Key words. Unsteady Stokes, linearized Navier-Stokes equations, boundary integral equations, fast algorithms.

AMS subject classifications. 33C10, 33F05, 35Q40, 35Q55

1. Introduction. In this paper, we consider integral equation formulations for time-dependent, linearized, viscous, incompressible flow (sometimes called unsteady Stokes flow or the linearized Navier-Stokes equations):

$$
\left\{\begin{aligned}
\frac{\partial \mathbf{u}}{\partial t} & =\Delta \mathbf{u}-\nabla p+\mathbf{g} \\
\nabla \cdot \mathbf{u} & =0
\end{aligned}\right.
$$

in a domain $D$ with smooth boundary $S$, subject to initial conditions

$$
\mathbf{u}(\mathbf{x}, 0)=\mathbf{u}_{0}(\mathbf{x}) \text { for } \mathbf{x} \in D .
$$

and either Dirichlet ("velocity") boundary conditions

$$
\mathbf{u}(\mathbf{x}, t)=\mathbf{f}(\mathbf{x}, t)
$$

or Neumann ("traction") boundary conditions

$$
\left(\frac{\partial \mathbf{u}_{i}(\mathbf{x}, t)}{\partial \mathbf{x}_{k}}+\frac{\partial \mathbf{u}_{k}(\mathbf{x}, t)}{\partial \mathbf{x}_{i}}-p(\mathbf{x}, t) \delta_{i k}\right) \mathbf{n}_{k}(\mathbf{x})=\mathbf{f}(\mathbf{x}, t)
$$

for $\mathbf{x} \in S, t \geq 0$.

In some settings, the forcing term $\mathbf{g}$ is absent, and in others it is a given function. The full incompressible Navier-Stokes equations can also be viewed as taking the form (1.1), with $\mathbf{g}=-\mathbf{u} \cdot \nabla \mathbf{u}$. Thus, new methods for the forced usteady Stokes equations have the potential for yielding improved methods for the general problem of incompressible flow, assuming only that the advective term $\mathbf{u} \cdot \nabla \mathbf{u}$ is treated explicitly.

\footnotetext{
*Department of Mathematical Sciences, New Jersey Institute of Technology, Newark, New Jersey 07102 (shidong.jiang@njit.edu).

${ }^{\dagger}$ Department of Mathematics, University of Michigan, Ann Arbor, Michigan 48109 (shravan@umich.edu).

${ }^{\ddagger}$ Courant Institute of Mathematical Sciences, New York University, New York, New York 10012 (greengard@courant.nyu.edu).
} 
Integral equation methods have been used for more than a century to establish existence and uniqueness results for a variety of elliptic, parabolic and hyperbolic partial differential equations (see, for example, [12, 37, 46, 49, 61, 62, 67, 74]). From a computational perspective, they have been used most extensively in the elliptic (steady state or time harmonic) case, because of their ability to handle complex geometry, unbounded domains and radiation conditions $[3,5,69]$ and because of the availability of fast algorithms to reduce the cost of handling the dense matrices that arise from their discretization. These algorithms include fast multipole methods, FFT or precorrected-FFT based methods, and hierarchical compression-based methods (wavelet and SVD-based schemes, H-matrices, HSS-matrices, etc.). The fundamental issue is that discretization of an elliptic boundary integral equation yields a dense $N \times N$ matrix, where $N$ denotes the number of degrees of freedom used to describe some unknown surface density on the given boundary. The straightforward application of a dense matrix to a vector requires $O\left(N^{2}\right)$ work, while classical Gaussian elimination techniques require $O\left(N^{3}\right)$ work to solve the system. The various fast algorithms listed above provide the ability to apply the discretized integral operator to a vector in $O(N)$ or $O(N \log N)$ operations. When combined with modern iterative methods (such as GMRES [70]), well-conditioned integral equation formulations have reduced the total work required to $O(N \log N)$, bringing large scale simulations within practical reach in electromagnetics, electrostatics, elasticity, fluid mechanics, and other application areas. Since the literature on this subject is vast, we simply refer the reader to a few relevant publications [11, 28, 29, 32, 33, 44, 54, 63, 66, 86, 87]. (For ill-conditioned problems or multiple right-hand sides, iterative methods are likely to be supplanted by fast direct solvers, which are now under intensive development $[7,8,25,57,27,39$, $40,42]$.)

In the case of parabolic or hyperbolic partial differential equations, integral equation methods have played an important role in analysis, especially for the isotropic heat, wave and Maxwell equations [5, 11, 10, 37, 48, 67, 60]. The numerical obstacles faced by integral equations in the time-dependent setting, however, are more severe. With $N_{T}$ time steps and $N_{S}$ points on the spatial boundary, direct evaluation of a space-time layer potential requires $O\left(N_{T}^{2} N_{S}^{2}\right)$ work, which is impractical even for problems of modest size. Because of their ability to handle complex, moving geometries and unbounded domains, however, substantial efforts have been made to improve efficiency. There now exist asymptotically fast algorithms for heat, acoustic, electromagnetic, and elastic wave potentials [31, 34, 35, 52, 60, 79, 81, 82].

We should note that time-domain integral equation methods offer several advantages over standard finite difference and/or finite element methods, aside from their geometric flexibility. First, for problems like (1.1), (1.2), (1.3), the unknowns are limited to the boundary $S$, since the forcing term and initial conditions can be accounted for analytically. This reduces the dimensionality of the problem by one and results in asymptotically optimal performance when combined with suitable fast algorithms. Second, it is easier to obtain high order accuracy in both space and time. Finally, in the case of unsteady flow, the divergence free constraint on the velocity field is incorporated into the representation itself. This avoids the need for either the solution of coupled field equations (as in fully implicit PDE-based marching schemes) or the projection of the velocity field onto divergence free fields at each time step (as in operator splitting methods).

The paper is organized as follows. In section 2 , we discuss the analytic properties of the governing Green's function, following the work of $[17,18,19,38,41,72,80]$. 
In section 3, we describe integral equation formulations for the pure initial problem, the inhomogeneous problem, the Dirichlet problem, and the Neumann problem. In section 4, a fast numerical algorithm is developed for solving the discretized integral equations, followed by a brief presentation of numerical results and a discussion of future work.

We note that Odqvist [64] first investigated the use of the double layer potential to prove existence of the solution to the Dirichlet problem for the unsteady Stokes flow in 1932. Fabes et al. [17, 18, 19] then studied the properties of the associated singular integral operators in detail. Subsequently, Shen [71] provided a careful proof of existence and uniqueness for the homogeneous (unforced) equations (1.1), (1.2), (1.3), in Lipschitz domains. A more elementary, but fairly comprehensive study of integral equation formulations for unsteady Stokes flow can be found in [43], which we follow more closely here.

2. Analytical preliminaries. We will use small boldfaced letters $(\mathbf{x}, \mathbf{y}, \mathbf{u}, \mathbf{v})$ to denote points (vectors) in $\mathbb{R}^{2}$. The magnitude of a vector $\mathbf{v}$ will be denoted by $\|\mathbf{v}\|$, the inner product of $\mathbf{u}$ and $\mathbf{v}$ by $\mathbf{u} \cdot \mathbf{v}$, and the tensor product of $\mathbf{u}$ and $\mathbf{v}$ by $\mathbf{u} \otimes \mathbf{v}$. We will have occasion to use the Fourier transform of a function in $\mathbb{R}^{2}$, and a point in the Fourier domain will be denoted by $\boldsymbol{\xi}$. We will use $t$ or $\tau$ to denote temporal variables. We will denote by $D$ a bounded domain in $\mathbb{R}^{2}$, and its boundary $\partial D$ by $S . S$ is assumed to be a smooth curve (at least twice differentiable). The outward unit normal vector at a point on $S$ will be denoted by $\mathbf{n}=\left(n_{1}, n_{2}\right)$. The space-time domain $D \times[0, T]$ will be denoted by $D_{T}$ and the space-time boundary $S \times[0, T]$ will be denoted by $S_{T}$. Since the Green's function for unsteady Stokes is a rather complicated tensor, we will make use of the Einstein summation convention when the context is clear.

2.1. The unsteady Stokeslet. Fundamental solutions for unsteady Stokes flow have been derived by several researchers (see, for example, [17, 18, 19, 38, 41, 65, 72, $80])$. The velocity field due to a unit force vector is called the unsteady Stokeslet (or Oseen's tensor) and denoted by $\mathbf{G}(\mathbf{x}, t ; \mathbf{y}, \tau)$, while the associated pressure field is called the pressurelet $\mathbf{p}(\mathbf{x}, t ; \mathbf{y}, \tau)$. They satisfy the equations

$$
\left\{\begin{aligned}
\frac{\partial \mathbf{G}}{\partial t} & =\Delta \mathbf{G}-\nabla \mathbf{p}+\delta(\mathbf{x}-\mathbf{y}) \delta(t-\tau) \mathbf{I} \\
\nabla \cdot \mathbf{G} & =0
\end{aligned}\right.
$$

where $\mathbf{I}$ is the $2 \times 2$ identity matrix and all the spatial derivatives are with respect to $\mathbf{x}$. Explicit expressions for $\mathbf{G}(\mathbf{x}, t ; \mathbf{y}, \tau)$ and $\mathbf{p}(\mathbf{x}, t ; \mathbf{y}, \tau)$ (see, for example, [38, 41, 80]) are given by the formulae

$$
\begin{gathered}
\mathbf{G}(\mathbf{x}, t ; \mathbf{y}, \tau)=\frac{e^{-\|\mathbf{r}\|^{2} / 4(t-\tau)}}{4 \pi(t-\tau)}\left(\mathbf{I}-\frac{\mathbf{r} \otimes \mathbf{r}}{\|\mathbf{r}\|^{2}}\right) \\
-\frac{1-e^{-\|\mathbf{r}\|^{2} / 4(t-\tau)}}{2 \pi\|\mathbf{r}\|^{2}}\left(\mathbf{I}-2 \frac{\mathbf{r} \otimes \mathbf{r}}{\|\mathbf{r}\|^{2}}\right) \\
\mathbf{p}(\mathbf{x}, t ; \mathbf{y}, \tau)=\frac{\mathbf{r}}{2 \pi\|\mathbf{r}\|^{2}} \delta(t-\tau)
\end{gathered}
$$

where $\mathbf{r}=\mathbf{x}-\mathbf{y}$ and the $\delta$ function is understood to satisfy $\int_{0}^{t} \delta(t-\tau) d \tau=1$. 
We define the associate stresslet $\mathbf{T}$ by the formula

$$
\mathbf{T}_{i j k}(\mathbf{x}, t ; \mathbf{y}, \tau)=\frac{\partial \mathbf{G}_{i j}(\mathbf{x}, t ; \mathbf{y}, \tau)}{\partial \mathbf{x}_{k}}+\frac{\partial \mathbf{G}_{k j}(\mathbf{x}, t ; \mathbf{y}, \tau)}{\partial \mathbf{x}_{i}}-\mathbf{p}_{j}(\mathbf{x}, t ; \mathbf{y}, \tau) \delta_{i k} .
$$

Note that $\mathbf{T}_{i j k}=\mathbf{T}_{k j i}$ as required by the symmetry of the stress tensor.

Another useful representation for the unsteady Stokeslet can be found in [17],

$$
\mathbf{G}(\mathbf{x}, t ; \mathbf{y}, \tau)=G_{H}(\mathbf{x}, t ; \mathbf{y}, \tau) \mathbf{I}+\mathbf{R} \otimes \mathbf{R}\left(G_{H}(\cdot, t-\tau)\right)(\mathbf{x}-\mathbf{y}),
$$

where $G_{H}$ is the fundamental solution of the heat equation

$$
G_{H}(\mathbf{x}, t ; \mathbf{y}, \tau)=G_{H}(\mathbf{x}-\mathbf{y}, t-\tau)=\frac{e^{-\frac{\|\mathbf{x}-\mathbf{y}\|^{2}}{4(t-\tau)}}}{4 \pi(t-\tau)},
$$

and the Riesz transform $\mathbf{R}$ in two dimensions is defined by the formula

$$
(\mathbf{R} f)(\mathbf{x})=\frac{1}{2 \pi} \mathbf{p} \cdot \mathbf{v} \cdot \int_{\mathbb{R}^{2}} \frac{\mathbf{x}-\mathbf{y}}{\|\mathbf{x}-\mathbf{y}\|^{3}} f(\mathbf{y}) d \mathbf{y}
$$

for a function $f$ defined on $\mathbb{R}^{2}$. (We have modified slightly the notation used in [17], following the definition of $\mathbf{R}$ in [75, 76]). It is, perhaps, worth noting that (2.5) is valid in higher dimensions, as well. In terms of the Fourier transform, the Riesz transform is given by

$$
\widehat{\mathbf{R} f}(\boldsymbol{\xi})=-\frac{i \boldsymbol{\xi}}{\|\boldsymbol{\xi}\|} \widehat{f}(\boldsymbol{\xi})
$$

Thus, we have

$$
\widehat{\mathbf{G}}(\boldsymbol{\xi}, t)=\widehat{G_{H}}(\boldsymbol{\xi}, t)\left(\mathbf{I}-\frac{\boldsymbol{\xi} \otimes \boldsymbol{\xi}}{\|\boldsymbol{\xi}\|^{2}}\right)
$$

and hence

$$
\mathbf{G}(\mathbf{x}, t ; \mathbf{y}, \tau)=\frac{1}{4 \pi^{2}} \int_{\mathbb{R}^{2}}\left(\mathbf{I}-\frac{\boldsymbol{\xi} \otimes \boldsymbol{\xi}}{\|\boldsymbol{\xi}\|^{2}}\right) e^{-\|\boldsymbol{\xi}\|^{2}(t-\tau)} e^{i(\mathbf{x}-\mathbf{y}) \cdot \boldsymbol{\xi}} d \boldsymbol{\xi}
$$

We also note that

$$
\mathbf{p}(\mathbf{x}, t ; \mathbf{y}, \tau)=-\nabla G_{L}(\mathbf{x}, \mathbf{y}) \delta(t-\tau),
$$

where $G_{L}=-\frac{1}{2 \pi} \ln \|\mathbf{x}-\mathbf{y}\|$ is the fundamental solution of the Laplace equation.

The following estimates for the unsteady Stokeslet can be derived from elementary arguments, following the treatment of the heat kernel in [24].

Lemma 2.1. For any fixed $t>\tau$ and $\mathbf{x} \in \mathbb{R}^{2}, \mathbf{G}_{i j}(\mathbf{x}, t ; \mathbf{y}, \tau)$ is bounded and absolutely integrable as a function of $\mathbf{y}$ over any compactly supported domain $D \in \mathbb{R}^{2}$. Moreover, for any $0<\alpha<1$,

$$
\left|\mathbf{G}_{i j}(\mathbf{x}, t ; \mathbf{y}, \tau)\right| \leq \frac{\text { const. }}{|t-\tau|^{\alpha}\|\mathbf{x}-\mathbf{y}\|^{2-2 \alpha}}
$$

Similarly, for any $1 / 2<\alpha<1$,

$$
\left|\frac{\partial \mathbf{G}_{i j}(\mathbf{x}, t ; \mathbf{y}, \tau)}{\partial \mathbf{y}_{k}}\right| \leq \frac{\text { const. }}{|t-\tau|^{\alpha}\|\mathbf{x}-\mathbf{y}\|^{3-2 \alpha}} .
$$


From the first estimate we have that, for a fixed $\mathbf{x} \in D, \mathbf{G}(\mathbf{x}, t ; \mathbf{y}, \tau)$ is absolutely integrable on $D_{T}=D \times[0, t]$ as a function of $\mathbf{y}$ and $\tau$. We also have that for a fixed $\mathbf{x} \in S=\partial D, \mathbf{G}(\mathbf{x}, t ; \mathbf{y}, \tau)$ is absolutely integrable on $S_{T}=S \times[0, t]$ as a function of $\mathbf{y}$ and $\tau$. From the second estimate, we conclude that for a fixed $\mathbf{x} \in D, \frac{\partial \mathbf{G}(\mathbf{x}, t ; \mathbf{y}, \tau)}{\partial \mathbf{y}_{k}}$ is absolutely integrable on $D_{T}$ as function of $\mathbf{y}$ and $\tau$.

2.2. Initial, volume, and layer potentials. The solution to the unsteady Stokes equations can be expressed as the superposition of an initial potential, a volume potential, and a combination of layer potentials. They are defined as follows:

Definition 2.2. Let $\mathbf{f}$ be a function defined on D. Then the initial potential operator $\mathcal{I}$ is defined by the formula

$$
\mathcal{I}[\mathbf{f}](\mathbf{x}, t)=\int_{D} \mathbf{G}(\mathbf{x}, t ; \mathbf{y}, 0) \mathbf{f}(\mathbf{y}) d \mathbf{y} .
$$

Definition 2.3. Let $\mathbf{g}$ be a function defined on $D_{T}$. Then the volume potential operator $\mathcal{V}$ is defined by the formula

$$
\mathcal{V}[\mathbf{g}](\mathbf{x}, t)=\int_{0}^{t} \int_{D} \mathbf{G}(\mathbf{x}, t ; \mathbf{y}, \tau) \mathbf{g}(\mathbf{y}, \tau) d \mathbf{y} d \tau .
$$

Definition 2.4. Let $\phi$ be a function defined on $S_{T}$. Then the single layer potential operator $\mathcal{S}$ is defined by the formula

$$
\mathcal{S}[\boldsymbol{\phi}](\mathbf{x}, t)=\int_{0}^{t} \int_{S} \mathbf{G}(\mathbf{x}, t ; \mathbf{y}, \tau) \phi(\mathbf{y}, \tau) d s(\mathbf{y}) d \tau .
$$

The double layer potential operator $\mathcal{D}$ is defined by the formula

$$
\mathcal{D}[\boldsymbol{\phi}](\mathbf{x}, t)=\int_{0}^{t} \int_{S} \mathbf{D}(\mathbf{x}, t ; \mathbf{y}, \tau) \phi(\mathbf{y}, \tau) d s(\mathbf{y}) d \tau,
$$

where the kernel of the double layer potential is given by

$$
\begin{aligned}
\mathbf{D}_{i j}(\mathbf{x}, t ; \mathbf{y}, \tau)= & \left(\frac{\partial \mathbf{G}_{i j}(\mathbf{x}, t ; \mathbf{y}, \tau)}{\partial \mathbf{y}_{k}}+\frac{\partial \mathbf{G}_{i k}(\mathbf{x}, t ; \mathbf{y}, \tau)}{\partial \mathbf{y}_{j}}\right) \mathbf{n}_{k}(\mathbf{y}) \\
+ & \mathbf{p}_{i}(\mathbf{x}, t ; \mathbf{y}, \tau) \mathbf{n}_{j}(\mathbf{y}) \\
=- & {\left[\left(\frac{\partial \mathbf{G}_{i j}(\mathbf{x}, t ; \mathbf{y}, \tau)}{\partial \mathbf{x}_{k}}+\frac{\partial \mathbf{G}_{i k}(\mathbf{x}, t ; \mathbf{y}, \tau)}{\partial \mathbf{x}_{j}}\right)\right.} \\
& \left.-\mathbf{p}_{i}(\mathbf{x}, t ; \mathbf{y}, \tau) \delta_{j k}\right] \mathbf{n}_{k}(\mathbf{y}) .
\end{aligned}
$$

The adjoint double layer potential operator $\Sigma$ is defined by the formula

$$
\Sigma[\phi](\mathbf{x}, t)=\int_{0}^{t} \int_{S} \boldsymbol{\sigma}(\mathbf{x}, t ; \mathbf{y}, \tau) \phi(\mathbf{y}, \tau) d s(\mathbf{y}) d \tau,
$$

where the kernel of the adjoint double layer potential is given by the formula

$$
\begin{aligned}
\boldsymbol{\sigma}_{i j}(\mathbf{x}, t ; \mathbf{y}, \tau)= & \mathbf{T}_{i j k}(\mathbf{x}, t ; \mathbf{y}, \tau) \mathbf{n}_{k}(\mathbf{x}) \\
=[ & {\left[\frac{\partial \mathbf{G}_{i j}(\mathbf{x}, t ; \mathbf{y}, \tau)}{\partial \mathbf{x}_{k}}+\frac{\partial \mathbf{G}_{k j}(\mathbf{x}, t ; \mathbf{y}, \tau)}{\partial \mathbf{x}_{i}}\right) } \\
& \left.-\mathbf{p}_{j}(\mathbf{x}, t ; \mathbf{y}, \tau) \delta_{i k}\right] \mathbf{n}_{k}(\mathbf{x}) .
\end{aligned}
$$


REMARK 2.5. Note that we have $\boldsymbol{\sigma}_{i j}(\mathbf{x}, t ; \mathbf{y}, \tau)=\mathbf{D}_{j i}(\mathbf{y}, t ; \mathbf{x}, \tau)$. That is, the adjointness of the operators is constructed with respect to the spatial variable, not the temporal variable.

Some properties of these potentials are listed below.

THEOREM 2.6.

1. For any $t>0$, the kernel of the initial potential operator is a smooth function. Thus, the initial potential operator $\mathcal{I}$ is a compact operator from $L^{2}(D)$ to itself.

2. The volume potential operator $\mathcal{V}$ is a compact operator from $L^{2}\left(D_{T}\right)$ to itself.

3. The single layer potential operator $\mathcal{S}$ is a compact operator from $L^{2}\left(S_{T}\right)$ to itself. The single layer potential $\mathcal{S}[\phi]$ is continuous across the boundary $S$.

4. The double layer potential operator $\mathcal{D}$ is a bounded (parabolically) singular integral operator from $L^{2}\left(S_{T}\right)$ to itself. The double layer potential $\mathcal{D}[\phi]$ satisfies the following jump relation

$$
\lim _{\epsilon \rightarrow 0+} \mathcal{D}[\phi](\mathbf{x} \pm \epsilon \mathbf{n}(\mathbf{x}), t)=\mathcal{D}[\phi](\mathbf{x}, t) \pm \frac{1}{2} \phi(\mathbf{x}, t), \quad \mathbf{x} \in S .
$$

5. The adjoint double layer potential operator $\Sigma$ is a bounded (parabolically) singular integral operator from $L^{2}\left(S_{T}\right)$ to itself. The adjoint double layer potential $\Sigma[\phi]$ satisfies the following jump relation

$$
\lim _{\epsilon \rightarrow 0+} \Sigma[\phi](\mathbf{x} \pm \epsilon \mathbf{n}(\mathbf{x}), t)=\Sigma[\phi](\mathbf{x}, t) \mp \frac{1}{2} \phi(\mathbf{x}, t), \quad \mathbf{x} \in S .
$$

Proof. The first three properties follow from Lemma 2.1. The facts that the double layer potential operator and its adjoint are bounded (parabolically) singular integral operators can be found in [18], along with an exposition of the theory of such operators. Detailed derivations of the jump relations for the double layer potential and its adjoint can be found in [43].

2.3. Green's formula for unsteady Stokes flow. Following the analysis for the heat equation [24], it is straightforward to establish a version of Green's formula.

THEOREM 2.7. Suppose that $\mathbf{u}, p$ satisfy the unsteady Stokes equations:

$$
\left\{\begin{aligned}
\frac{\partial \mathbf{u}}{\partial t} & =\Delta \mathbf{u}-\nabla p+\mathbf{g}(\mathbf{x}, t), \quad(\mathbf{x}, t) \in D_{T} \\
\nabla \cdot \mathbf{u} & =0, \quad(\mathbf{x}, t) \in D_{T}, \\
\mathbf{u}(\mathbf{x}, 0) & =\mathbf{u}_{0}(\mathbf{x}), \quad \mathbf{x} \in D, \quad t=0
\end{aligned}\right.
$$

Then

$$
\begin{aligned}
& \int_{0}^{t} \int_{S} \mathbf{G}(\mathbf{x}, t ; \mathbf{y}, \tau) \mathbf{f}(\mathbf{y}, \tau) d s(\mathbf{y}) d \tau-\int_{0}^{t} \int_{S} \mathbf{D}(\mathbf{x}, t ; \mathbf{y}, \tau) \mathbf{u}(\mathbf{y}, \tau) d s(\mathbf{y}) d \tau \\
& +\int_{D} \mathbf{G}(\mathbf{x}, t ; \mathbf{y}, 0) \mathbf{u}_{0}(\mathbf{y}) d \mathbf{y}+\int_{0}^{t} \int_{D} \mathbf{G}(\mathbf{x}, t ; \mathbf{y}, \tau) \mathbf{g}(\mathbf{y}, \tau) d \mathbf{y} d \tau \\
& = \begin{cases}\mathbf{u}(\mathbf{x}, t), & \mathbf{x} \in D, \\
\frac{1}{2} \mathbf{u}(\mathbf{x}, t), & \mathbf{x} \in S, \\
0, & \mathbf{x} \notin \bar{D},\end{cases}
\end{aligned}
$$


where

$$
\begin{aligned}
\mathbf{f}_{j}(\mathbf{y}, \tau) & =\left(\frac{\partial \mathbf{u}_{j}(\mathbf{y}, \tau)}{\partial \mathbf{y}_{k}}+\frac{\partial \mathbf{u}_{k}(\mathbf{y}, \tau)}{\partial \mathbf{y}_{j}}\right) \mathbf{n}_{k}(\mathbf{y})-p(\mathbf{y}, \tau) \mathbf{n}_{j}(\mathbf{y}) \\
& =\left(\frac{\partial \mathbf{u}_{j}(\mathbf{y}, \tau)}{\partial \mathbf{y}_{k}}+\frac{\partial \mathbf{u}_{k}(\mathbf{y}, \tau)}{\partial \mathbf{y}_{j}}-p(\mathbf{y}, \tau) \delta_{j k}\right) \mathbf{n}_{k}(\mathbf{y})
\end{aligned}
$$

is the jth component of the surface force.

Proof. See [43] $\square$

Note that the four terms on the left side of (2.28) correspond to a single layer potential, a double layer potential, an initial potential, and a volume potential, respectively. This formula is the basis for the "direct" boundary integral approach. That is, if velocity boundary conditions are specified, one can solve a boundary integral equation for the unknown $\mathbf{f}$, while if traction (force) boundary conditions are specified, one can solve an integral equation for the boundary values of $\mathbf{u}$.

3. Indirect integral equation formulations for unsteady Stokes flow. While the direct boundary integral formulation yields a second kind Volterra equation for force/traction boundary conditions, the same is not true for velocity boundary conditions, which lead to a first kind integral equation. To obtain a well-conditioned equation, we will make use of abstract layer potentials whose densities are unknown functions, with no immediate physical interpretation. First, however, we will use the fact that the Stokes equations are linear and reduce the general problem to one with zero inital and forcing data.

Theorem 3.1. (Unsteady Stokes flow in free-space). Suppose that $\mathbf{u}_{\mathbf{0}} \in C^{2}\left(\mathbb{R}^{2}\right)$ is a divergence-free vector field compactly supported in the domain $D \subset \mathbb{R}^{2}$ and that $\mathrm{g} \in C_{1}^{2}\left(\mathbb{R}^{2} \times[0, \infty)\right)$, also compactly supported in $D$. Then the solution to the problem (2.27) is given by the formulae

$$
\begin{aligned}
& \mathbf{u}^{(I F)}(\mathbf{x}, t)=\mathcal{I}\left[\mathbf{u}_{0}\right](\mathbf{x}, t)+\mathcal{V}[\mathbf{g}](\mathbf{x}, t) \\
& p^{(I F)}(\mathbf{x}, t)=\int_{0}^{t} \int_{\mathbb{R}^{2}} \mathbf{p}(\mathbf{x}, t ; \mathbf{y}, \tau) \cdot \mathbf{g}(\mathbf{y}, \tau) d \mathbf{y} d \tau+h(t) .
\end{aligned}
$$

Furthermore, $\mathcal{I}\left[\mathbf{u}_{0}\right](\mathbf{x}, t) \in C_{\infty}^{2}\left(\mathbb{R}^{2} \times(0, \infty)\right), \mathcal{V}[\mathbf{g}](\mathbf{x}, t) \in C_{1}^{2}\left(\mathbb{R}^{2} \times(0, \infty)\right)$, and $p^{(I F)} \in$ $C_{1}^{2}\left(\mathbb{R}^{2} \times(0, \infty)\right)$. The proof of this theorem can be found in [43]. Note that the representation (3.1) is an explicit integral transform using known data, not an integral equation. No linear system of equations needs to be solved.

3.1. The Dirichlet problem. Let us now consider the Dirichlet problem, where velocity boundary conditions are specified: (1.1), (1.2), (1.3). We seek to represent the solution in the form

$$
\begin{aligned}
& \mathbf{u}(\mathbf{x}, t)=\mathbf{u}^{(I F)}(\mathbf{x}, t)+\mathbf{u}^{(B)}(\mathbf{x}, t) \\
& p(\mathbf{x}, t)=p^{(I F)}(\mathbf{x}, t)+p^{(B)}(\mathbf{x}, t)
\end{aligned}
$$

where $\mathbf{u}^{(I F)}, p^{(I F)}$ are defined in $(3.1)$ and $\mathbf{u}^{(B)}, p^{(B)}$ constitute a solution to the unsteady Stokes equations with zero initial and forcing data. 
The following theorem states that we may represent $\mathbf{u}^{(B)}, p^{(B)}$ using a double layer potential, resulting in a second kind boundary integral equation for an unknown density.

Theorem 3.2. Suppose that $D$ is a bounded domain and that $\mathbf{f}$ is a continuous function on $S_{T}$, satisfying the following compatibility conditions

$$
\begin{aligned}
\mathbf{f}(\mathbf{x}, 0) & =\mathbf{u}_{0}(\mathbf{x}), \quad \mathbf{x} \in S, \\
\int_{S} \mathbf{f}(\mathbf{y}, t) \cdot \mathbf{n}(\mathbf{y}) d s(\mathbf{y}) & =0, \quad t \in[0, T]
\end{aligned}
$$

Suppose further that the velocity $\mathbf{u}^{(B)}$ and the pressure $p^{(B)}$ are represented via the formulae

$$
\begin{aligned}
\mathbf{u}^{(B)}(\mathbf{x}, t) & =\int_{0}^{t} \int_{S} \mathbf{D}(\mathbf{x}, t ; \mathbf{y}, \tau) \phi(\mathbf{y}, \tau) d s(\mathbf{y}) d \tau, \\
p^{(B)}(\mathbf{x}, t) & =\int_{S} G_{L}(\mathbf{x}, \mathbf{y}) \frac{\partial \phi(\mathbf{y}, t)}{\partial t} \cdot \mathbf{n}(\mathbf{y}) d s(\mathbf{y}) \\
& +2 \int_{S} \frac{\partial^{2} G_{L}(\mathbf{x}, \mathbf{y})}{\partial \mathbf{y}_{j} \partial \mathbf{y}_{k}} \mathbf{n}_{k}(\mathbf{y}) \phi_{j}(\mathbf{y}, t) d s(\mathbf{y}),
\end{aligned}
$$

respectively, where the kernel $\mathbf{D}$ is defined in (2.18). Then (3.3) is the solution to the pure Dirichlet problem

$$
\begin{aligned}
\frac{\partial \mathbf{u}^{(B)}(\mathbf{x}, t)}{\partial t} & =\Delta \mathbf{u}^{(B)}(\mathbf{x}, t)-\nabla p^{(B)}(\mathbf{x}, t), \quad(\mathbf{x}, t) \in D_{T} \\
\nabla \cdot \mathbf{u}^{(B)} & =0, \quad(\mathbf{x}, t) \in D_{T} \\
\mathbf{u}^{(B)}(\mathbf{x}, 0) & =\mathbf{0}, \quad \mathbf{x} \in D \\
\mathbf{u}^{(B)}(\mathbf{x}, t) & =\mathbf{f}(\mathbf{x}, t)-\mathbf{u}^{(I F)}(\mathbf{x}, t), \quad(\mathbf{x}, t) \in S_{T}
\end{aligned}
$$

if the unknown density $\phi$ satisfies the equation

$$
-\frac{1}{2} \phi(\mathbf{x}, t)+\int_{0}^{t} \int_{S} \mathbf{D}(\mathbf{x}, t ; \mathbf{y}, \tau) \phi(\mathbf{y}, \tau) d s(\mathbf{y}) d \tau=\mathbf{f}(\mathbf{x}, t)-\mathbf{u}^{(I F)}(\mathbf{x}, t)
$$

for $(\mathbf{x}, t) \in S_{T}$. The proof of the above theorem can be found in [43].

Note that the original Dirichlet data $\mathbf{f}(\mathbf{x}, t)$ has been replaced by $\mathbf{f}(\mathbf{x}, t)-\mathbf{u}^{(I F)}(\mathbf{x}, t)$ so that $\mathbf{u}=\mathbf{u}^{(I F)}+\mathbf{u}^{(B)}$ satisfies the desired boundary condition. If $D$ were an unbounded (exterior) domain, then the boundary integral equations would have the form

$$
\frac{1}{2} \phi(\mathbf{x}, t)+\int_{0}^{t} \int_{S} \mathbf{D}(\mathbf{x}, t ; \mathbf{y}, \tau) \phi(\mathbf{y}, \tau) d s(\mathbf{y}) d \tau=\mathbf{f}(\mathbf{x}, t)-\mathbf{u}^{(I F)}(\mathbf{x}, t) .
$$

3.2. The Neumann problem. When force/traction conditions are specified, we will make use of an abstract single layer potential, obtaining an integral equation involving the adjoint double layer potential. That is, in order to solve: (1.1), (1.2), (1.4), we again represent the solution in the form

$$
\begin{aligned}
& \mathbf{u}(\mathbf{x}, t)=\mathbf{u}^{(I F)}(\mathbf{x}, t)+\mathbf{u}^{(B)}(\mathbf{x}, t) \\
& p(\mathbf{x}, t)=p^{(I F)}(\mathbf{x}, t)+p^{(B)}(\mathbf{x}, t),
\end{aligned}
$$


where $\mathbf{u}^{(B)}, p^{(B)}$ is a solution to the unsteady Stokes equations with zero initial and forcing data.

THEOREM 3.3. Suppose that $D$ is a bounded domain and that the velocity $\mathbf{u}^{(B)}$ and pressure $p^{(B)}$ are represented by the formulae

$$
\begin{aligned}
\mathbf{u}^{(B)}(\mathbf{x}, t) & =\int_{0}^{t} \int_{S} \mathbf{G}(\mathbf{x}, t ; \mathbf{y}, \tau) \phi(\mathbf{y}, \tau) d s(\mathbf{y}) d \tau \\
p^{(B)}(\mathbf{x}, t) & =\int_{0}^{t} \int_{S} \mathbf{p}(\mathbf{x}, t ; \mathbf{y}, \tau) \cdot \boldsymbol{\phi}(\mathbf{y}, \tau) d s(\mathbf{y}) d \tau \\
& =\int_{S} \nabla_{\mathbf{y}} G_{L}(\mathbf{x}, \mathbf{y}) \cdot \boldsymbol{\phi}(\mathbf{y}, \tau) d s(\mathbf{y}),
\end{aligned}
$$

respectively. Then (3.7) is the solution to the pure Neumann problem

$$
\begin{aligned}
\frac{\partial \mathbf{u}(\mathbf{x}, t)}{\partial t} & =\Delta \mathbf{u}(\mathbf{x}, t)-\nabla p(\mathbf{x}, t), \quad(\mathbf{x}, t) \in D_{T} \\
\nabla \cdot \mathbf{u} & =0, \quad(\mathbf{x}, t) \in D_{T} \\
\mathbf{u}(\mathbf{x}, 0) & =\mathbf{0}, \quad \mathbf{x} \in D \\
\left(\frac{\partial \mathbf{u}_{i}(\mathbf{x}, t)}{\partial \mathbf{x}_{k}}+\frac{\partial \mathbf{u}_{k}(\mathbf{x}, t)}{\partial \mathbf{x}_{i}}-p(\mathbf{x}, t) \delta_{i k}\right) & \mathbf{n}_{k}(\mathbf{x}) \\
& =\mathbf{f}(\mathbf{x}, t)-\mathbf{f}^{(I F)}(\mathbf{x}, t), \quad(\mathbf{x}, t) \in S_{T}
\end{aligned}
$$

where

$$
\mathbf{f}^{(I F)}(\mathbf{x}, t)=\left(\frac{\partial \mathbf{u}_{i}^{(I F)}(\mathbf{x}, t)}{\partial \mathbf{x}_{k}}+\frac{\partial \mathbf{u}_{k}^{(I F)}(\mathbf{x}, t)}{\partial \mathbf{x}_{i}}-p^{(I F)}(\mathbf{x}, t) \delta_{i k}\right) \mathbf{n}_{k}(\mathbf{x}),
$$

if the unknown density $\phi$ satisfies the equation

$$
\frac{1}{2} \phi(\mathbf{x}, t)+\int_{0}^{t} \int_{S} \boldsymbol{\sigma}(\mathbf{x}, t ; \mathbf{y}, \tau) \phi(\mathbf{y}, \tau) d s(\mathbf{y}) d \tau=\mathbf{f}(\mathbf{x}, t)-\mathbf{f}^{(I F)}(\mathbf{x}, t),
$$

for $(\mathbf{x}, t) \in S_{T}$, where $\boldsymbol{\sigma}$ is defined in $(2.22)$.

The proof of the above theorem can be found in [43]. If $D$ were an exterior domain, the boundary integral equation would take the form

$$
-\frac{1}{2} \phi(\mathbf{x}, t)+\int_{0}^{t} \int_{S} \boldsymbol{\sigma}(\mathbf{x}, t ; \mathbf{y}, \tau) \phi(\mathbf{y}, \tau) d s(\mathbf{y}) d \tau=\mathbf{f}(\mathbf{x}, t)-\mathbf{f}^{(I F)}(\mathbf{x}, t)
$$

for $(\mathbf{x}, t) \in S_{T}$.

3.3. Nullspaces of the boundary integral equations. It was shown in [71] that the boundary integral equation (3.9) for the interior Neumann problem has no nontrivial nullspace, and therefore a unique solution. Since the boundary integral equation (3.6) for the exterior Dirichlet problem is the adjoint of (3.9), it has a unique solution as well. For the exterior Neumann problem, an argument similar to that of [71] can be used to shown that the nullspace of the associated boundary integral equation (3.10) consists of any function of the form $f(t) \mathbf{n}(\mathbf{x})$, where $f \in L^{2}[0, T]$ and $\mathbf{n}(\mathbf{x})$ is the unit outward normal vector. This, in turn, indicates that the boundary integral equation (3.5) for the interior Dirichlet problem has a nullspace consisting of functions of the form $f(t) \eta(\mathbf{x})$, where $f \in L^{2}[0, T]$ for some vector-valued function $\eta(\mathbf{x})$. 
4. Discretization and Numerical Evaluation of Layer Potentials. For the sake of brevity, we will limit our presentation to the discretization and evaluation of layer potentials, with a brief discussion in the conclusions concerning initial and volume potentials. In other words, for the remainder of this paper, we are largely concerned with the solution of the problems (3.4) and (3.8). (The initial and volume potentials contribute to $\mathbf{u}^{(I F)}$ but are known functions.) For the sake of simplicity, we will also assume that the boundary is stationary. Unlike with finite difference or finite element methods, it is straightforward to design high order schemes in the integral equation context, especially when the governing operators are second kind Volterra or Fredholm operators. In that setting, the order of accuracy of the scheme is simply the order of accuracy of the underlying quadrature rule. Here, we present quadratures sufficient for 4th order accuracy in time and couple it with a sixteenth order scheme in space. Higher order multistep schemes can be derived by analogy, although for order greater than six or so, it is better not to use equal size time steps. Instead, one can construct better-conditioned multi-stage marching schemes using composite Chebyshev or Legendre grids (see, for example, [81]).

4.1. Discretization and evaluation of the single layer potential. We first consider the evaluation of the single layer potential, following the treatment of the heat equation in $[31,35,53]$. There are three fundamental observations to be made. First, we can split the single layer potential $\mathcal{S}[\phi]$ defined in (2.16) into two parts - a local part and a history part:

$$
\mathcal{S}[\phi]=\mathcal{S}_{L}[\phi]+\mathcal{S}_{H}[\phi],
$$

where the local part $\mathcal{S}_{L}$ is defined by the formula

$$
\mathcal{S}_{L}[\boldsymbol{\phi}](\mathbf{x}, t)=\int_{t-\delta}^{t} \int_{S} \mathbf{G}(\mathbf{x}, t ; \mathbf{y}, \tau) \boldsymbol{\phi}(\mathbf{y}, \tau) d \tau d s(\mathbf{y}),
$$

and the history part $\mathcal{S}_{H}$ is defined by the formula

$$
S_{H}[\boldsymbol{\phi}](\mathbf{x}, t)=\int_{0}^{t-\delta} \int_{S} \mathbf{G}(\mathbf{x}, t ; \mathbf{y}, \tau) \phi(\mathbf{y}, \tau) d s(\mathbf{y}) d \tau .
$$

Second, the Stokes kernels are sharply peaked at $\mathbf{y}=\mathbf{x}$ and singular as $\tau$ approaches $t$, which will require some care in quadrature. Third, when $\tau$ is bounded away from the current time $t$, as in the history part, then the kernels (and resulting fields) are smooth and admit a variety of simpler approximations.

In order to overcome the singular quadrature issues, we design special product integration-based schemes, following the treatment in [53]. In that paper, it is shown that for robustness, it is essential to interchange the order of integration in the layer potentials and to carry out integration in time analytically for polynomial approximations of the layer potential density $\phi(\mathbf{y}, \tau)$.

It is straightforward to verify the following interpolation result.

Lemma 4.1. Let $f(\tau)$ denote a smooth function on the interval $[t, t-(k-1) \Delta t]$ for $k \geq 2$. Then

$$
f(\tau)=\left[\begin{array}{llll}
1 & \frac{t-\tau}{\Delta t} & \ldots & \frac{(t-\tau)^{k-1}}{\Delta t^{k-1}}
\end{array}\right] C_{k}\left[\begin{array}{c}
f(t) \\
f(t-\Delta t) \\
\vdots \\
f(t-(k-1) \Delta t)
\end{array}\right]+O\left(\Delta t^{k}\right),
$$


where the coefficient matrices $C_{k}$ are given by the formulas:

$$
C_{k}=\left\{\begin{array}{lll}
{\left[\begin{array}{cc}
1 & 0 \\
-1 & 1
\end{array}\right],} & & k=2 \\
{\left[\begin{array}{ccc}
1 & 0 & 0 \\
-\frac{3}{2} & 2 & -\frac{1}{2} \\
\frac{1}{2} & -1 & \frac{1}{2}
\end{array}\right],} & k=3 \\
{\left[\begin{array}{cccc}
1 & 0 & 0 & 0 \\
-\frac{11}{6} & 3 & -\frac{3}{2} & \frac{1}{3} \\
1 & -\frac{5}{2} & 2 & -\frac{1}{2} \\
-\frac{1}{6} & \frac{1}{2} & -\frac{1}{2} & \frac{1}{6}
\end{array}\right],} & k=4
\end{array}\right.
$$

4.1.1. Evaluation of the local part. Suppose now that the density $\phi(y, \tau)$ in $\mathcal{S}_{L}$ is approximated by a polynomial of degree $k-1$ with respect to time (for each fixed $\mathbf{y})$ :

$$
\phi(\mathbf{y}, \tau)=\left[\begin{array}{llll}
1 & \frac{t-\tau}{\Delta t} & \ldots & \frac{(t-\tau)^{k-1}}{\Delta t^{k-1}}
\end{array}\right] C_{k}\left[\begin{array}{c}
\phi(\mathbf{y}, t) \\
\phi(\mathbf{y}, t-\Delta t) \\
\vdots \\
\phi(\mathbf{y}, t-(k-1) \Delta t)
\end{array}\right]+O\left(\Delta t^{k}\right)
$$

Exact integration using this approximation will yield $k$ th order accuracy for the layer potential, using the standard product integration formalism. That is, we substitute (2.2) and (4.6) into (4.2) and integrate in time analytically. The result is

$$
\mathcal{S}_{L}[\phi](\mathbf{x}, t)=\int_{S}\left[\begin{array}{llll}
\mathbf{G}_{0} & \frac{\mathbf{G}_{1}}{\Delta t} & \cdots & \frac{\mathbf{G}_{k-1}}{\Delta t^{k-1}}
\end{array}\right] C_{k}\left[\begin{array}{c}
\phi(\mathbf{y}, t) \\
\phi(\mathbf{y}, t-\Delta t) \\
\vdots \\
\phi(\mathbf{y}, t-(k-1) \Delta t)
\end{array}\right] d s(\mathbf{y})+O\left(\Delta t^{k}\right),
$$

where the kernels $\mathbf{G}_{j}(j=0, \cdots, k-1)$ are defined by the formula

$$
\mathbf{G}_{j}(\mathbf{x}, \mathbf{y})=\frac{1}{4 \pi}\left(\mathbf{I}-\frac{\mathbf{r} \otimes \mathbf{r}}{\|\mathbf{r}\|^{2}}\right) \mathbf{G}_{j}^{1}-\frac{1}{8 \pi}\left(\mathbf{I}-2 \frac{\mathbf{r} \otimes \mathbf{r}}{\|\mathbf{r}\|^{2}}\right) \mathbf{G}_{j}^{2},
$$


with the functions $\mathbf{G}_{j}^{1}$ and $\mathbf{G}_{j}^{2}$ defined by the formulas

$$
\begin{gathered}
\mathbf{G}_{j}^{1}=\int_{t-\delta}^{t} e^{-\|\mathbf{r}\|^{2} / 4(t-\tau)}(t-\tau)^{j-1} d \tau \\
=\left(\frac{\|\mathbf{r}\|^{2}}{4}\right)^{j} \int_{\rho}^{\infty} \frac{e^{-\lambda}}{\lambda^{j+1}} d \lambda \quad\left(\lambda=\frac{\|\mathbf{r}\|^{2}}{4(t-\tau)}\right) \\
= \begin{cases}\frac{4}{\|\mathbf{r}\|^{2}} e^{-\rho}, & j=-1, \\
E_{1}(\rho), & j=0, \\
\delta\left(e^{-\rho}-\rho E_{1}(\rho),\right. & j=1, \\
\frac{\delta^{2}}{2}\left((1-\rho) e^{-\rho}+\rho^{2} E_{1}(\rho)\right), & j=2, \\
\frac{\delta^{3}}{6}\left(\left(2-\rho+\rho^{2}\right) e^{-\rho}-\rho^{3} E_{1}(\rho)\right), & j=3 .\end{cases} \\
\mathbf{G}_{j}^{2}=\int_{t-\delta}^{t} \frac{1-e^{-\lambda}(t-\tau)^{j-1} d \tau}{\lambda} \\
=\left(\begin{array}{ll}
\left.\frac{\|\mathbf{r}\|^{2}}{4}\right)^{j} \int_{\rho}^{\infty} \frac{1-e^{-\lambda}}{\lambda^{j+2}} d \lambda & j=0, \\
\frac{1-e^{-\rho}+E_{1}(\rho),}{\rho} & j=1, \\
\frac{\delta}{2}\left(\frac{1-e^{-\rho}}{\rho}+e^{-\rho}-\rho E_{1}(\rho)\right), & j=3 . \\
\frac{\delta^{2}}{6}\left(2 \frac{1-e^{-\rho}}{\rho}+(1-\rho) e^{-\rho}+\rho^{2} E_{1}(\rho)\right), & \left.j=\rho^{3} E_{1}(\rho)\right), \\
\frac{\delta^{3}}{24}\left(6 \frac{1-e^{-\rho}}{\rho}+\left(2-\rho+\rho^{2}\right) e^{-\rho}\right. & j=1
\end{array}\right.
\end{gathered}
$$

Here $\rho=\frac{\|\mathbf{x}-\mathbf{y}\|^{2}}{4 \delta}$, and $E_{1}(x)=\int_{x}^{\infty} \frac{e^{-t}}{t} d t$ is the exponential integral function (see, for example, [1]).

REMARK 4.2. The kernel $\mathbf{G}_{-1}^{1}$ is not needed for the single layer potential, but it is needed for the evaluation of the double layer potential and included here for completeness.

Since the exponential integral function is logarithmically singular at the origin, we can use the quadratures in $[2,45]$ to discretize each of the spatial integrals in $(4.5)$ with high-order accuracy. Moreover, since the kernels are all smooth in the far field, they are suitable for the application of a variety of fast summation algorithms, such as the kernel independent fast multipole method $[22,26,58,84,85,88])$. The total computational cost for the local part is therefore $O\left(N_{S} N_{T}\right)$ where $N_{S}$ is the number of discretization points on the boundary $S$ and $N_{T}$ is the number of time steps.

4.1.2. Evaluation of the history part. For the history part, we first observe that (2.10) gives a Fourier integral representation for the kernel $\mathbf{G}(\mathbf{x}, t ; \mathbf{y}, \tau)$. Note that the integrand decays to zero exponentially fast when $t-\tau \geq \delta$ is bounded away from 0. In [31], it was shown that an efficient sum-of-exponentials approximation for the heat kernel exists in this case. Exactly the same idea can be applied here, with 
the result that $N_{F} \approx 1 / \delta$ discrete Fourier modes are need to satisfy an estimate of the form

$$
\left|\mathbf{G}(\mathbf{x}, t ; \mathbf{y}, \tau)-\frac{1}{4 \pi^{2}} \sum_{j=1}^{N_{F}} w_{j}\left(\mathbf{I}-\frac{\boldsymbol{\xi}_{j} \otimes \boldsymbol{\xi}_{j}}{\left\|\boldsymbol{\xi}_{j}\right\|^{2}}\right) e^{-\left\|\boldsymbol{\xi}_{j}\right\|^{2}(t-\tau)} e^{i(\mathbf{x}-\mathbf{y}) \cdot \boldsymbol{\xi}_{j}}\right| \leq \epsilon,
$$

where $\boldsymbol{\xi}_{j}$ and $w_{j}\left(j=1, \cdots, N_{F}\right)$ are the quadrature nodes and weights, respectively. The estimate (4.11) holds uniformly for all $t-\tau \geq \delta>0$ and $\|\mathbf{x}\|,\|\mathbf{y}\| \leq R$ for some fixed value of $R$.

REMARK 4.3. In practice, the construction of [31], which uses tensor product quadrature on dyadically scaled intervals can be improved through the use of generalized Gaussian quadrature $[6,83,55]$. The number of Fourier modes needed depends on $\delta$, $R$, and the desired precision $\epsilon$. For example, we need 21600 Fourier modes to achieve 13-digit accuracy for $\delta=10^{-3}$ and $R=1$.

REMARK 4.4. For interior problems, one could use any of a variety of Green's functions which satisfy the defining equations (2.1) within the computational domain D. For example, we could use the periodic unsteady Stokeslet on a larger box, as in the original fast algorithm for the heat equation [35]. In that case, the discretized Fourier integral discretization is replaced with a truncated Fourier series representation.

Substituting (4.11) into (4.3) and rearranging terms, we see that the history part $\mathcal{S}_{H}$ can be written as follows:

$$
\mathcal{S}_{H}[\boldsymbol{\phi}](\mathbf{x}, t) \approx \frac{1}{4 \pi^{2}} \sum_{j=1}^{N_{F}} w_{j}\left(\mathbf{I}-\frac{\boldsymbol{\xi}_{j} \otimes \boldsymbol{\xi}_{j}}{\left\|\boldsymbol{\xi}_{j}\right\|^{2}}\right) e^{i \mathbf{x} \cdot \boldsymbol{\xi}_{j}} H_{j}\left(\boldsymbol{\xi}_{j}, t\right)
$$

where the history modes $H_{j}\left(j=1, \cdots, N_{F}\right)$ are defined by the formula

$$
H_{j}\left(\boldsymbol{\xi}_{j}, t\right)=\int_{0}^{t-\delta} e^{-\left\|\boldsymbol{\xi}_{j}\right\|^{2}(t-\tau)} F_{j}(\tau) d \tau
$$

with

$$
F_{j}(\tau)=\int_{S} e^{-i \boldsymbol{\xi}_{j} \cdot \mathbf{y}} \boldsymbol{\phi}(\mathbf{y}, \tau) d s(\mathbf{y})
$$

For each fixed $\tau$, the integral in (4.14) can be discretized using the trapezoidal rule, that is

$$
F_{j}(\tau) \approx \frac{L_{S}}{N_{S}} \sum_{l=1}^{N_{S}} e^{-i \boldsymbol{\xi}_{j} \cdot \mathbf{y}_{l}} \phi\left(\mathbf{y}_{l}, \tau\right) s\left(\mathbf{y}_{l}\right)
$$

with $L_{S}$ the total length of the boundary $S, s\left(\mathbf{y}_{l}\right)$ the Jacobian at $\mathbf{y}_{l}$, and $N_{S}$ the number of discretization points on the boundary $S$. The trapezoidal rule is spectrally accurate since the integrand is smooth and periodic and the sum can be computed rapidly using the nonuniform Fast Fourier Transform (NUFFT) (see, for example, $[4,14,15,21,23,30,50,68])$. The total computational cost for evaluating $F_{j}$ for $j=1, \cdots, N_{F}$ at $N_{T}$ time steps is $O\left(N_{T}\left(N_{F}+N_{S}\right) \log \left(N_{F}+N_{S}\right)\right)$.

The reader may have noticed that we replaced the history-dependent part $\mathcal{S}_{H}[\phi](\mathbf{x}, t)$ with what appears, at first glance, to be an even more complicated history-dependent representation, namely (4.12). Once the $F_{j}$ have been computed, however, the history 
modes $H_{j}\left(\boldsymbol{\xi}_{j}, i \delta\right)$ can be seen to satisfy a simple recurrence relation [31, 35, 52]. More precisely, for a $k$ th-order multistep schemes, we have

$$
\begin{aligned}
H_{j}\left(\boldsymbol{\xi}_{j}, t\right) & =\int_{0}^{t-\delta} e^{-\left\|\boldsymbol{\xi}_{j}\right\|^{2}(t-\tau)} F_{j}(\tau) d \tau \\
& =e^{-(k-1)\left\|\boldsymbol{\xi}_{j}\right\|^{2} \Delta t} H_{j}\left(\boldsymbol{\xi}_{j}, t-(k-1) \Delta t\right) \\
& +e^{-\left\|\boldsymbol{\xi}_{j}\right\|^{2} \delta} \int_{t-\delta-(k-1) \Delta t}^{t-\delta} e^{-\left\|\boldsymbol{\xi}_{j}\right\|^{2}(t-\delta-\tau)} F_{j}(\tau) d \tau .
\end{aligned}
$$

We need to be careful, however, in computing the last integral on the right-hand side of (4.16) because we are nearing the singular point $\tau=t$. We rely, again, on product integration.

For a $k$ th-order scheme, we approximate $F_{j}(\tau)$ by an interplation polynomial of degree $k-1$

$$
F_{j}(\tau)=\left[\begin{array}{llll}
1 & \frac{t-\delta-\tau}{\Delta t} & \ldots & \frac{(t-\delta-\tau)^{k-1}}{\Delta t^{k-1}}
\end{array}\right] C_{k}\left[\begin{array}{c}
F_{j}(t-\delta) \\
F_{j}(t-\delta-\Delta t) \\
\vdots \\
F_{j}(t-\delta-(k-1) \Delta t)
\end{array}\right]+O\left(\Delta t^{k}\right) .
$$

Substituting (4.17) into (4.16) and integrating analytically, we obtain

$$
\begin{aligned}
& \int_{t-\delta-(k-1) \Delta t}^{t-\delta} e^{-\left\|\boldsymbol{\xi}_{j}\right\|^{2}(t-\delta-\tau)} F_{j}(\tau) d \tau \\
& =\left[\begin{array}{llll}
W_{j}^{0} & W_{j}^{1} & \cdots & W_{j}^{k-1}
\end{array}\right] C_{k}\left[\begin{array}{c}
F_{j}(t-\delta) \\
F_{j}(t-\delta-\Delta t) \\
\vdots \\
F_{j}(t-\delta-(k-1) \Delta t)
\end{array}\right]+O\left(\Delta t^{k+1}\right),
\end{aligned}
$$

where the weights $W_{j}^{i}(i=0, \cdots, k-1)$ are given by the formulas

$$
\begin{array}{rlr}
W_{j}^{i} & =\frac{1}{\Delta t^{i}} \int_{t-\delta-(k-1) \Delta t}^{t-\delta} e^{-\left\|\boldsymbol{\xi}_{j}\right\|^{2}(t-\delta-\tau)}(t-\delta-\tau)^{i} d \tau & \\
& =\frac{1}{\Delta t^{i}} \int_{0}^{(k-1) \Delta t} e^{-\left\|\boldsymbol{\xi}_{j}\right\|^{2} x} x^{i} d x, \quad(x=t-\delta-\tau) & i=0, \\
& =(k-1)^{i+1} \Delta t \cdot \begin{cases}\frac{1-e^{-\beta}}{\beta}, & i=1, \\
\frac{1-e^{-\beta}-\beta e^{-\beta}}{\beta^{2}}, & i=2, \\
\frac{2-2 e^{-\beta}-2 \beta e^{-\beta}-\beta^{2} e^{-\beta}}{\beta^{3}}, & i=3, \\
\frac{6-6 e^{-\beta}-6 \beta e^{-\beta}-3 \beta^{2} e^{-\beta}-\beta^{3} e^{-\beta}}{\beta^{4}}, & i=3\end{cases}
\end{array}
$$

where $\beta=(k-1)\left\|\boldsymbol{\xi}_{j}\right\|^{2} \Delta t$. 
The preceding formulas are subject to catastrophic cancellation when $\beta$ is small. They can be computed accurately, however, by replacing them with their Taylor expansions. For $\beta<10^{-3}$, we use

$$
W_{j}^{i}=(k-1)^{i+1} \Delta t \cdot \begin{cases}1-\frac{1}{2} \beta+\frac{1}{6} \beta^{2}-\frac{1}{24} \beta^{3}+\frac{1}{120} \beta^{4}, & i=0, \\ \frac{1}{2}-\frac{1}{3} \beta+\frac{1}{8} \beta^{2}-\frac{1}{30} \beta^{3}+\frac{1}{144} \beta^{4}, & i=1, \\ \frac{1}{3}-\frac{1}{4} \beta+\frac{1}{10} \beta^{2}-\frac{1}{36} \beta^{3}+\frac{1}{168} \beta^{4}, & i=2, \\ \frac{1}{4}-\frac{1}{5} \beta+\frac{1}{12} \beta^{2}-\frac{1}{42} \beta^{3}+\frac{1}{192} \beta^{4}, & i=3,\end{cases}
$$

which is accurate to about 12 digits.

If $N_{F}=O\left(N_{S}\right)$, then the total computational cost for the evaluation of the history part is $O\left(N_{T} N_{S} \log N_{S}\right)$. This is possible, for example, when taking large time steps with $\delta=\Delta t=\Delta x$, where $\Delta x$ is the mesh spacing on the the boundary $S$.

4.2. Evaluation of the double layer potential. The evaluation of the double layer potential is analogous to that of the single layer potential, with minor changes in the nature of the kernels for the local part and a slightly different Fourier representation for the history part. As before, we split the double layer potential $\mathcal{D}[\phi]$ into a local part $\mathcal{D}_{L}$ and a history part $\mathcal{D}_{H}$ :

$$
\begin{aligned}
\mathcal{D}[\phi] & =\mathcal{D}_{L}[\phi]+\mathcal{D}_{H}[\phi] \\
& :=\int_{t-\delta}^{t} \int_{S} \mathbf{D}(\mathbf{x}, t ; \mathbf{y}, \tau) \phi(\mathbf{y}, \tau) d \tau d s(\mathbf{y}) \\
& +\int_{0}^{t-\delta} \int_{S} \mathbf{D}(\mathbf{x}, t ; \mathbf{y}, \tau) \phi(\mathbf{y}, \tau) d s(\mathbf{y}) d \tau .
\end{aligned}
$$

In more detail, we first write the double layer kernel as follows:

$$
\begin{aligned}
\mathbf{D}(\mathbf{x}, t ; \mathbf{y}, \tau) & =\frac{\mathbf{n}(\mathbf{y}) \otimes \mathbf{r}+(\mathbf{n}(\mathbf{y}) \cdot \mathbf{r})\left(\mathbf{I}-2 \frac{\mathbf{r} \otimes \mathbf{r}}{\|\mathbf{r}\|^{2}}\right)}{8 \pi} \frac{e^{-\lambda}}{(t-\tau)^{2}}+\frac{\mathbf{r} \otimes \mathbf{n}(\mathbf{y})}{2 \pi\|\mathbf{r}\|^{2}} \delta(t-\tau) \\
& -\frac{\mathbf{n}(\mathbf{y}) \otimes \mathbf{r}+\mathbf{r} \otimes \mathbf{n}(\mathbf{y})+(\mathbf{n}(\mathbf{y}) \cdot \mathbf{r})\left(\mathbf{I}-4 \frac{\mathbf{r} \otimes \mathbf{r}}{\|\mathbf{r}\|^{2}}\right)}{8 \pi} \frac{1-e^{-\lambda}-\lambda e^{-\lambda}}{\lambda^{2}(t-\tau)^{2}}
\end{aligned}
$$

where $\lambda=\frac{\|\mathbf{r}\|^{2}}{4(t-\tau)}$. As above, this leads to an approximation of the form:

$$
\mathcal{D}_{L}[\phi](\mathbf{x}, t)=\int_{S}\left[\begin{array}{llll}
\mathbf{D}_{0} & \frac{\mathbf{D}_{1}}{\Delta t} & \cdots & \frac{\mathbf{D}_{k-1}}{\Delta t^{k-1}}
\end{array}\right] C_{k}\left[\begin{array}{c}
\phi(\mathbf{y}, t) \\
\phi(\mathbf{y}, t-\Delta t) \\
\vdots \\
\phi(\mathbf{y}, t-(k-1) \Delta t)
\end{array}\right] d s(\mathbf{y})+O\left(\Delta t^{k}\right),
$$

where the kernels $\mathbf{D}_{j}(j=0, \cdots, k-1)$ are defined by the formula

$$
\begin{aligned}
\mathbf{D}_{j}(\mathbf{x}, \mathbf{y}) & =\frac{\mathbf{n}(\mathbf{y}) \otimes \mathbf{r}+(\mathbf{n}(\mathbf{y}) \cdot \mathbf{r})\left(\mathbf{I}-2 \frac{\mathbf{r} \otimes \mathbf{r}}{\|\mathbf{r}\|^{2}}\right)}{8 \pi} \mathbf{D}_{j}^{1} \\
& -\frac{\mathbf{n}(\mathbf{y}) \otimes \mathbf{r}+\mathbf{r} \otimes \mathbf{n}(\mathbf{y})+(\mathbf{n}(\mathbf{y}) \cdot \mathbf{r})\left(\mathbf{I}-4 \frac{\mathbf{r} \otimes \mathbf{r}}{\|\mathbf{r}\|^{2}}\right)}{8 \pi} \mathbf{D}_{j}^{2}+\frac{\mathbf{r} \otimes \mathbf{n}(\mathbf{y})}{2 \pi\|\mathbf{r}\|^{2}} \delta_{j 0}
\end{aligned}
$$


with the functions $\mathbf{D}_{j}^{1}$ and $\mathbf{D}_{j}^{2}$ defined by the formulas

$$
\begin{aligned}
& \mathbf{D}_{j}^{1}=\int_{t-\delta}^{t} e^{-\|\mathbf{r}\|^{2} / 4(t-\tau)}(t-\tau)^{j-2} d \tau \\
& =\mathbf{G}_{j-1}^{1} \text {. } \\
& \mathbf{D}_{j}^{2}=\int_{t-\delta}^{t} \frac{1-e^{-\lambda}-\lambda e^{-\lambda}}{\lambda^{2}(t-\tau)^{2}}(t-\tau)^{j} d \tau \\
& =\left(\frac{\|\mathbf{r}\|^{2}}{4}\right)^{j-1} \int_{\rho}^{\infty} \frac{1-e^{-\lambda}-\lambda e^{-\lambda}}{\lambda^{j+2}} d \lambda \\
& = \begin{cases}\frac{4}{\|\mathbf{r}\|^{2}} \frac{1-e^{-\rho}}{\rho}, & j=0, \\
\frac{1}{2}\left(\frac{1-e^{-\rho}-\rho e^{-\rho}}{\rho^{2}}+E_{1}(\rho)\right), & j=1, \\
\frac{\delta}{3}\left(\frac{1-e^{-\rho}-\rho e^{-\rho}}{\rho^{2}}+e^{-\rho}-\rho E_{1}(\rho)\right), & j=2, \\
\frac{\delta^{2}}{8}\left(2 \frac{1-e^{-\rho}-\rho e^{-\rho}}{\rho^{2}}+(1-\rho) e^{-\rho}+\rho^{2} E_{1}(\rho)\right), & j=3 .\end{cases}
\end{aligned}
$$
tion.

Here $\rho=\frac{\|\mathbf{x}-\mathbf{y}\|^{2}}{4 \delta}$, and $E_{1}(x)=\int_{x}^{\infty} \frac{e^{-t}}{t} d t$ is again the exponential integral func-

For the history part $\mathcal{D}_{H}[\phi]$, we will need the Fourier representation of the double layer kernel. Differentiating (2.10) with respect to $\mathbf{y}$, we obtain

$$
\begin{aligned}
& \tilde{\mathbf{D}}(\mathbf{x}, t ; \mathbf{y}, \tau)=\frac{-i}{4 \pi^{2}} \int_{\mathbb{R}^{2}} {\left[(\mathbf{n}(\mathbf{y}) \cdot \boldsymbol{\xi})\left(\mathbf{I}-2 \frac{\boldsymbol{\xi} \otimes \boldsymbol{\xi}}{\|\boldsymbol{\xi}\|^{2}}\right)+\mathbf{n}(\mathbf{y}) \otimes \boldsymbol{\xi}\right] } \\
& \cdot e^{-\|\boldsymbol{\xi}\|^{2}(t-\tau)} e^{i(\mathbf{x}-\mathbf{y}) \cdot \boldsymbol{\xi}} d \boldsymbol{\xi},
\end{aligned}
$$

where $\tilde{\mathbf{D}}(\mathbf{x}, t ; \mathbf{y}, \tau)$ is the double layer potential kernel without the pressure part $((2.18))$. Pressure does not contribute to the history part, since the pressurelet $\mathbf{p}$ is instantaneous and contributes only to the velocity at the current time $t$. We use exactly the same quadrature nodes and weights as in (4.11) to discretize (4.27) and obtain an efficient sum-of-exponentials approximation for $\tilde{\mathbf{D}}(\mathbf{x}, t ; \mathbf{y}, \tau)$ :

$$
\begin{aligned}
& \tilde{\mathbf{D}}(\mathbf{x}, t ; \mathbf{y}, \tau) \approx \frac{-i}{4 \pi^{2}} \sum_{j=1}^{N_{F}} w_{j} {\left[\left(\mathbf{n}(\mathbf{y}) \cdot \boldsymbol{\xi}_{j}\right)\left(\mathbf{I}-2 \frac{\boldsymbol{\xi}_{j} \otimes \boldsymbol{\xi}_{j}}{\left\|\boldsymbol{\xi}_{j}\right\|^{2}}\right)+\mathbf{n}(\mathbf{y}) \otimes \boldsymbol{\xi}_{j}\right] } \\
& \cdot e^{-\left\|\boldsymbol{\xi}_{j}\right\|^{2}(t-\tau)} e^{i(\mathbf{x}-\mathbf{y}) \cdot \boldsymbol{\xi}_{j}}
\end{aligned}
$$

Substituting the above equation into the expression for the history part, we obtain

$$
\mathcal{D}_{H}[\boldsymbol{\phi}](\mathbf{x}, t) \approx \frac{-i}{4 \pi^{2}} \sum_{j=1}^{N_{F}} w_{j} e^{i \mathbf{x} \cdot \boldsymbol{\xi}_{j}} H_{j}^{d}\left(\boldsymbol{\xi}_{j}, t\right),
$$

where the history modes $H_{j}^{d}\left(j=1, \cdots, N_{F}\right)$ are defined by the formula

$$
H_{j}^{d}\left(\boldsymbol{\xi}_{j}, t\right)=\int_{0}^{t-\delta} e^{-\left\|\boldsymbol{\xi}_{j}\right\|^{2}(t-\tau)} F_{j}^{d}(\tau) d \tau
$$


with

$$
F_{j}^{d}(\tau)=\int_{S} e^{-i \boldsymbol{\xi}_{j} \cdot \mathbf{y}}\left[\left(\mathbf{n}(\mathbf{y}) \cdot \boldsymbol{\xi}_{j}\right)\left(\mathbf{I}-2 \frac{\boldsymbol{\xi}_{j} \otimes \boldsymbol{\xi}_{j}}{\left\|\boldsymbol{\xi}_{j}\right\|^{2}}\right)+\mathbf{n}(\mathbf{y}) \otimes \boldsymbol{\xi}_{j}\right] \boldsymbol{\phi}(\mathbf{y}, \tau) d s(\mathbf{y}) .
$$

The rest of the procedure for evaluating the history part of the double layer potential is entirely analogous to that for the single layer.

REMARK 4.5. The evaluation of the adjoint double layer potential $\Sigma[\phi]$ is similar. The necessary formulas are obtained easily from the symmetry between the kernels $\mathbf{D}$ and $\boldsymbol{\sigma}$ (see Remark 2.5).

4.3. Treatment of initial and volume data. When either the initial or forcing data are nonzero, we need to compute the solution $\mathbf{u}^{(I F)}$. For short times, the fast Gauss transform can be used to evaluate the initial potential and for longer times, the initial potential can be folded into the (smooth) history part. The volume potential can be treated by a decomposition into local and history parts as well, relying on the same fast algorithms as above.

4.4. Solution of the integral equation. With the machinery for evaluating layer potentials in place, we turn to the solution of the Dirichlet problem (3.4), using the integral equation (3.5). At time $t$, note that all previous values of the density $(\phi(\mathbf{y}, t-\Delta t), \phi(\mathbf{y}, t-2 \Delta t), \phi(\mathbf{y}, t-3 \Delta t), \ldots)$ have already been computed.

For the $k$ th order scheme, we need to solve the following boundary integral equation for $\phi(\mathbf{x}, t)$

$$
\begin{aligned}
& -\frac{1}{2} \phi(\mathbf{x}, t)+\int_{S} \sum_{j=0}^{k-1} \frac{\mathbf{D}_{j}}{\Delta t^{j}} C_{k}^{j, 1} \phi(\mathbf{y}, t) d s(\mathbf{y}) \\
& =-\int_{S} \sum_{l=2}^{k} \sum_{j=0}^{k-1} \frac{\mathbf{D}_{j}}{\Delta t^{j}} C_{k}^{j, l} \phi(\mathbf{y}, t-(l-1) \Delta t) d s(\mathbf{y})-\mathcal{D}_{H}[\boldsymbol{\phi}]+\mathbf{f}(\mathbf{x}, t)
\end{aligned}
$$

where $C_{k}^{j, l}$ is the $(j, l)$ entry of the interpolatory coefficient matrix given in (4.5) and $\mathbf{f}$ is the given Dirichlet data. Note that the terms on the right side of (4.32) are known at the current time $t$.

THEOREM 4.6. For any $k \geq 2$, the boundary integral equation (4.32) is a second kind Fredholm equation with a one-dimensional nullspace.

Proof. Close inspection shows that $\mathbf{D}_{j}(\mathbf{x}, \mathbf{y})$ defined in (4.24) $(j \geq 1)$ involves $E_{1}(\rho), \frac{1-e^{-\rho}-\rho e^{-\rho}}{\rho^{2}}$, and other smooth terms. The exponential integral function $E_{1}$ is logarithmically singular at the origin (see, for example, [1]), and a simple calculation shows that $\lim _{\rho \rightarrow 0} \frac{1-e^{-\rho}-\rho e^{-\rho}}{\rho^{2}}=\frac{1}{2}$. Therefore, the kernels $\mathbf{D}_{j}$ are at most weakly singular (and integrable) for $j \geq 1$.

Thus, we only need to consider $\mathbf{D}_{0}$. Combining (4.9), (4.24)- (4.26), we obtain

$$
\begin{aligned}
\mathbf{D}_{0}(\mathbf{x}, \mathbf{y}) & =\frac{\mathbf{n}(\mathbf{y}) \otimes \mathbf{r}+(\mathbf{n}(\mathbf{y}) \cdot \mathbf{r})\left(\mathbf{I}-2 \frac{\mathbf{r} \otimes \mathbf{r}}{\|\mathbf{r}\|^{2}}\right)}{2 \pi\|\mathbf{r}\|^{2}} e^{-\rho} \\
& -\frac{\mathbf{n}(\mathbf{y}) \otimes \mathbf{r}+\mathbf{r} \otimes \mathbf{n}(\mathbf{y})+(\mathbf{n}(\mathbf{y}) \cdot \mathbf{r})\left(\mathbf{I}-4 \frac{\mathbf{r} \otimes \mathbf{r}}{\|\mathbf{r}\|^{2}}\right)}{2 \pi\|\mathbf{r}\|^{2}} \frac{1-e^{-\rho}}{\rho}+\frac{\mathbf{r} \otimes \mathbf{n}(\mathbf{y})}{2 \pi\|\mathbf{r}\|^{2}} .
\end{aligned}
$$

Since $e^{-\rho}-\frac{1-e^{-\rho}}{\rho}=O(\rho)=O\left(\|\mathbf{r}\|^{2}\right)$ and $1-\frac{1-e^{-\rho}}{\rho}=O(\rho)=O\left(\|\mathbf{r}\|^{2}\right)$, it is easy to see that terms involving $\mathbf{n}(\mathbf{y}) \otimes \mathbf{r}$ and $\mathbf{r} \otimes \mathbf{n}(\mathbf{y})$ are both bounded. Furthermore, a 
classical result in potential theory states that $\mathbf{n}(\mathbf{y}) \cdot \mathbf{r}$ is $O\left(\|\mathbf{r}\|^{2}\right)$ when both $\mathbf{x}$ and $\mathbf{y}$ are on the boundary. Thus the terms involving $\mathbf{n}(\mathbf{y}) \cdot \mathbf{r}$ are also bounded. It follows from the above facts that $\mathbf{D}_{0}$ is bounded for $\mathbf{x}, \mathbf{y} \in S$. Thus, the kernel in (4.32) is at most weakly singular and the corresponding operator is compact, which implies that (4.32) is a system of second kind Fredholm equations.

Since the fully time-dependent boundary integral equation (3.5) for the interior Dirichlet problem has a nullspace consisting of functions of the form $f(t) \eta(\mathbf{x})$ for any $f \in L^{2}([0, T])$ and a fixed function $\eta$, it is easy to see that this implies that the semidiscretized equation (4.32) has a one-dimensional nullspace spanned by $\eta$ alone.

口

In order to solve (4.32) numerically, we first add a rank-one term to the left-hand side as follows:

$$
\begin{aligned}
& -\frac{1}{2} \boldsymbol{\phi}(\mathbf{x}, t)+\int_{S} \sum_{j=0}^{k-1} \frac{\mathbf{D}_{j}}{\Delta t^{j}} C_{k}^{j, 1} \boldsymbol{\phi}(\mathbf{y}, t) d s(\mathbf{y}) \\
& -\alpha \mathbf{z}(\mathbf{x}) \int_{S} \mathbf{n}(\mathbf{y}) \cdot \boldsymbol{\phi}(\mathbf{y}, k \delta) d s(\mathbf{y}) \\
& =-\int_{S} \sum_{l=2}^{k} \sum_{j=0}^{k-1} \frac{\mathbf{D}_{j}}{\Delta t^{j}} C_{k}^{j, l} \phi(\mathbf{y}, t-(l-1) \Delta t) d s(\mathbf{y})-\mathcal{D}_{H}[\boldsymbol{\phi}]+\mathbf{f}(\mathbf{x}, t),
\end{aligned}
$$

where $\alpha$ is a nonzero constant and $\mathbf{z}$ is an arbitrary function satifying the constraint

$$
\int_{S} \mathbf{n}(\mathbf{y}) \cdot \mathbf{z}(\mathbf{y}) d s(\mathbf{y})=1
$$

One convenient choice for $\mathbf{z}$ is $\mathbf{n} / L_{S}$, where $L_{S}$ is the total arclength of the boundary $S$. This modification is discuss in detail in [69] (page 123). The modified equation always has a unique solution and its solution satisfies the original equation (4.32) so long as the right-hand side satisfies the compatibility condition (i.e., that the integral of the normal component of the right hand side over $S$ is zero).

In short, (4.34) is now a second kind integral equation with a trivial nullspace and can therefore be solved efficiently using iterative solvers like GMRES. A good starting vector for the iterative solver is simply the solution at the previous step.

REMARK 4.7. For stationary boundaries (and a fixed $\Delta t$ ), we need to solve a linear system with the same governing matrix but a different right hand side at each time step. In that setting, one can achieve even faster solution times by using fast direct solvers as described in [27, 42, 56, 57]. That is, one can construct a sparse representation for the inverse matrix (once) and simply apply it to the right-hand side vector when marching in time.

REMARK 4.8. The spectrum of the discretized system plays a critical role in the stability of the overall scheme. Using the quadrature scheme in [2] to discretize (4.34) and the fourth order interpolation scheme in time, we have not observed any instability. We have not, however, carried out a detailed analysis of this issue.

The solution of the Neumann problem is nearly identical, differing only in the use of the adjoint kernels in the integral operators, as discussed in Remark 4.5). For 
the interior Neumann problem, the rank-one modification is not needed since it has a trivial nullspace. The modification is necessary for the exterior Neumann case.

5. Numerical examples. In this section, we illustrate the performance of the integral equation method in the case of the pure Dirichlet problem, where $\mathbf{u}^{(I F)}=0$. We consider four simple boundary curves: a circle, an ellipse with aspect ratio 2:1, a crescent, and a smooth hexagram, shown in Figures 1-4, respectively. The final time is set to $T=10$. Each domain is of approximately unit area. We use 64 spatial discretization points for the circle, 128 points for the ellipse and crescent, and 256 points for the hexagram. We use a sixteenth-order accurate quadrature for logarithmically singular integrands [2], so that the accuracy is dominated by the discretization error in time.

In each of the following tables, $\Delta t$ is the time step; $K$ is the condition number of the linear system that needs to be solved at each time step; $p$ is the average number of iterations of GMRES at each time step (we restart GMRES every 10 iterations and set the tolerance to $10^{-12}$ ); $E$ is the relative $L^{2}$ error of the numerical solution at the final time; and $r$ is the ratio of relative $L^{2}$ errors on successive time step refinements - that is, $r(j)=E(j-1) / E(j)$. The last column displays the total CPU time.

REMARK 5.1. We construct exact solutions by placing unsteady Stokeslet sources randomly outside the computational domain.

\begin{tabular}{cccccc}
\hline$\Delta t$ & $K$ & $p$ & $E$ & $r$ & $\begin{array}{c}\text { CPU } \\
\text { time }\end{array}$ \\
\hline $2.00 \mathrm{e} 0$ & 1.97 & 16.0 & $1.44 \mathrm{e}-3$ & & $6.24 \mathrm{e}-1$ \\
$1.00 \mathrm{e} 0$ & 1.94 & 16.4 & $1.41 \mathrm{e}-4$ & 10.2 & $1.52 \mathrm{e} 0$ \\
$5.00 \mathrm{e}-1$ & 1.90 & 16.8 & $5.90 \mathrm{e}-6$ & 24.0 & $2.70 \mathrm{e} 0$ \\
$2.50 \mathrm{e}-1$ & 1.85 & 17.4 & $4.76 \mathrm{e}-7$ & 12.4 & $5.48 \mathrm{e} 0$ \\
$1.25 \mathrm{e}-1$ & 1.84 & 17.6 & $3.30 \mathrm{e}-8$ & 14.4 & $1.03 \mathrm{e} 1$ \\
$6.25 \mathrm{e}-2$ & 1.94 & 17.8 & $2.28 \mathrm{e}-9$ & 14.5 & $2.31 \mathrm{e} 1$ \\
\hline
\end{tabular}

FIG. 5.1. Numerical results for a circle geometry.

\begin{tabular}{cccccc}
\hline$\Delta t$ & $K$ & $p$ & $E$ & $r$ & $\begin{array}{c}\text { CPU } \\
\text { time }\end{array}$ \\
\cline { 2 - 6 } $2.00 \mathrm{e} 0$ & 4.95 & 25.0 & $1.87 \mathrm{e}-3$ & & $1.48 \mathrm{e} 0$ \\
$1.00 \mathrm{e} 0$ & 4.78 & 25.1 & $1.65 \mathrm{e}-4$ & 11.4 & $2.47 \mathrm{e} 0$ \\
$5.00 \mathrm{e}-1$ & 4.53 & 25.0 & $4.06 \mathrm{e}-6$ & 40.5 & $4.48 \mathrm{e} 0$ \\
$2.50 \mathrm{e}-1$ & 4.30 & 25.3 & $3.29 \mathrm{e}-7$ & 12.4 & $8.52 \mathrm{e} 0$ \\
$1.25 \mathrm{e}-1$ & 4.14 & 26.5 & $2.52 \mathrm{e}-8$ & 13.1 & $1.66 \mathrm{e} 1$ \\
$6.25 \mathrm{e}-2$ & 4.02 & 25.4 & $1.93 \mathrm{e}-9$ & 13.0 & $3.49 \mathrm{e} 1$ \\
\hline
\end{tabular}

FIG. 5.2. Numerical results for a 2-1 ellipse geometry.

Several observations about Figures (5.1)-(5.4) are in order. First, there is no stability issue in taking large time steps. Indeed, the largest time step in our simulation is 2 . Second, the linear systems which need to be solved have very modest condition numbers, which are more or less independent of the time step. Third, the convergence rates are consistent with fourth order accuracy. Finally, the total CPU time is roughly proportional to the number of time steps.

6. Conclusions. We have presented well-conditioned second kind integral equation formulations for two standard boundary value problems governed by the unsteady 


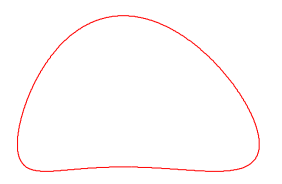

\begin{tabular}{cccccc}
\hline$\Delta t$ & $K$ & $p$ & $E$ & $r$ & $\begin{array}{c}\text { CPU } \\
\text { time }\end{array}$ \\
\hline $2.00 \mathrm{e} 0$ & 5.51 & 30.0 & $4.91 \mathrm{e}-3$ & & $1.33 \mathrm{e} 0$ \\
$1.00 \mathrm{e} 0$ & 5.25 & 30.0 & $3.09 \mathrm{e}-4$ & 15.9 & $2.38 \mathrm{e} 0$ \\
$5.00 \mathrm{e}-1$ & 4.94 & 29.9 & $4.92 \mathrm{e}-6$ & 62.9 & $4.35 \mathrm{e} 0$ \\
$2.50 \mathrm{e}-1$ & 4.65 & 29.3 & $3.12 \mathrm{e}-7$ & 15.7 & $9.41 \mathrm{e} 0$ \\
$1.25 \mathrm{e}-1$ & 4.48 & 29.2 & $1.46 \mathrm{e}-8$ & 21.3 & $1.76 \mathrm{e} 1$ \\
$6.25 \mathrm{e}-2$ & 4.37 & 28.5 & $1.07 \mathrm{e}-9$ & 13.7 & $3.55 \mathrm{e} 1$ \\
\hline
\end{tabular}

FIG. 5.3. Numerical results for a crescent geometry.

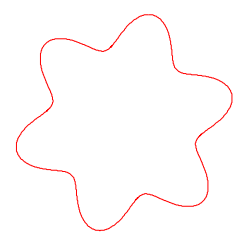

\begin{tabular}{cccccc}
\hline$\Delta t$ & $K$ & $p$ & $E$ & $r$ & $\begin{array}{c}\text { CPU } \\
\text { time }\end{array}$ \\
\hline $2.00 \mathrm{e} 0$ & 14.2 & 44.0 & $1.23 \mathrm{e}-3$ & & $2.18 \mathrm{e} 0$ \\
$1.00 \mathrm{e} 0$ & 13.5 & 43.3 & $8.01 \mathrm{e}-5$ & 15.3 & $4.41 \mathrm{e} 0$ \\
$5.00 \mathrm{e}-1$ & 12.7 & 41.4 & $2.56 \mathrm{e}-6$ & 31.3 & $7.78 \mathrm{e} 0$ \\
$2.50 \mathrm{e}-1$ & 11.8 & 38.0 & $2.00 \mathrm{e}-7$ & 12.8 & $1.60 \mathrm{e} 1$ \\
$1.25 \mathrm{e}-1$ & 10.7 & 38.8 & $1.31 \mathrm{e}-8$ & 15.3 & $3.14 \mathrm{e} 1$ \\
$6.25 \mathrm{e}-2$ & 9.77 & 36.9 & $8.7 \mathrm{e}-10$ & 15.1 & $6.73 \mathrm{e} 1$ \\
\hline
\end{tabular}

FIG. 5.4. Numerical results for a smooth hexagram geometry.

Stokes equations. For either the Dirichlet problem (velocity boundary conditions) or the Neumann problem (force/traction boundary conditions), this involves explicit initial and volume potentials and the solution of an integral equation for an unknown density on the domain boundary. (In the absence of boundary conditions, the solution is explicit and involves only the initial and volume potentials with known data.)

We have also described numerical methods based on these integral representations and demonstrated that high order accuracy is straightforward to achieve (although the corresponding quadratures are somewhat technical). Though our current implementation is linear in time and quadratic in space, the numerical algorithms we presented here can be carried out in optimal (or near optimal) time using suitable fast algorithms, which we have only sketched out here. We have restricted our attention mainly to the analytic foundations of the method and the order of accuracy in time.

The integral equation formulations and associated algorithms are easily generalized to the three dimensional case. An important advantage of our approach is that it can be extended to moving and free boundary problems as well as a variety of interface problems. Finally, as noted in the introduction, the forced unsteady Stokes problem is a driver for solvers of the full incompressible Navier-Stokes equation, assuming only that the advective term is treated explicitly. These applications are currently under investigation.

Acknowledgments. S. Jiang was supported in part by the National Science Foundation under grant CCF-0905395. S. Veerapaneni was supported in part by startup funds from the University of Michigan. L. Greengard was supported in part by the Applied Mathematical Sciences Program of the U.S. Department of Energy under Contract DEFGO288ER25053.

\section{REFERENCES}

[1] M. Abramowitz And I. A. Stegun, Handbook of Mathematical Functions, Dover, 1965. 
[2] B. K. Alpert, Hybrid Gauss-trapezoidal quadrature rules, SIAM J. Sci. Comput., 20 (1999), $1551-1584$.

[3] P. K. Banerjee And R. Butterfield, Boundary element methods in engineering science, McGraw-Hill, 1981.

[4] G. Beylkin, On the fast Fourier transform of functions with singularities, Appl. Comput. Harmonic Anal., 2 (1995), 363-383.

[5] C. A. Brebbia, J. C. F. Telles, and L. C. Wrobel, Boundary element techniques, SpringerVerlag, 1984.

[6] J. Bremer, Z. Gimbutas, And V. Rokhlin, A nonlinear optimization procedure for generalized Gaussian quadratures, SIAM J. Sci. Comput., 32 (2010), 1761-1788.

[7] S. Chandrasekaran, P. Dewilde, M. Gu, W. Lyons, and T. Pals, A fast solver for HSS representations via sparse matrices, SIAM J Matrix Anal. Appl., 29 (2006), 67-81.

[8] S. Chandrasekaran, M. Gu, and T. Pals, A fast $U L V$ decomposition solver for hierarchically semiseparable representations, SIAM J Matrix Anal. Appl., 28 (2006), 603-622.

[9] H. Cheng, L. Greengard, and V. Rokhlin, A fast adaptive multipole algorithm in three dimensions, J. Comput. Phys., 155 (1999), 468-498.

[10] M. Costabel, Time-dependent problems with the boundary integral equation method, Chapter 25, Encyclopedia of Computational Mechanics, (E. Stein, R. De Borst, T.J.R. Hughes, eds), John Wiley, 2004.

[11] W. C. Chew, J.-M. Jin, E. Michielssen, And J. Song, eds. Fast and Efficient Algorithms in Computational Electromagnetics, Artech House, Boston, 2001.

[12] D. Colton And R. KRess, Integral equation methods in scattering theory, Wiley-Interscience Publication, New York, 1983.

[13] M. G. Duffy, Quadrature over a pyramid or cube of integrands with a singularity at a vertex, SIAM J. Num. Anal., 19 (1982), 1260-1262.

[14] A. Dutt and V. Rokhlin, Fast Fourier transforms for nonequispaced data, SIAM J. Sci. Comput., 14 (1993), 1368-1393.

[15] A. Dutt and V. Rokhlin, Fast Fourier transforms for nonequispaced data. II, Appl. Comput. Harmon. Anal., 2 (1995), 85-100.

[16] L. C. Evans, Partial differential equations, AMS, 1998.

$[17]$ E. B. Fabes, B. F. Jones, And N. M. Riviere, The initial value problem for the Navier-Stokes equations with data in $L^{p}$, Arch. Rational Mech. Anal., 45 (1972), pp. 222-240.

[18] E. B. Fabes, J. E. Lewis, AND N. M. Riviere, Singular Integrals and Hydrodynamic Potentials, Am. Jour. of Math., 99 (1977), 601-625.

[19] E. B. Fabes, J. E. Lewis, AND N. M. Riviere, Boundary value problems for the Navier-Stokes Equations, Am. Jour. of Math., 99 (1977), 626-668.

[20] E. B. Fabes, C. E. Kenig, and G. C. Verchota, The Dirichlet Problem for the Stokes System on Lipschitz Domains, Duke Math. J., 57 (1988), 769-793.

[21] J.A. Fessler And B.P. SutTon, Nonuniform fast Fourier transforms using minmax interpolatio, IEEE Trans. Signal Proc., 51 (2003), 560-574.

[22] W. Fong And E. Darve, The black-box fast multipole method, J. Comput. Phys., 228 (2009), $8712-8725$.

[23] K. FOURMONT, Non-equispaced fast fourier transforms with applications to tomography, IEEE Trans. Signal Proc., 9 (2003), 431-450.

[24] A. Friedman, Partial differential equations of parabolic type, Prentice Hall, 1964.

[25] A. Gillman, P. Young, and P.G. Martinsson, A direct solver with $O(N)$ complexity for integral equations on one-dimensional domains, submitted.

[26] Z. Gimbutas and V. Rokhlin, A generalized fast multipole method for nonoscillatory kernels, SIAM J. Sci. Comput., 24 (2002), 796-817.

[27] L. Greengard, D. Gueyffier, P.G. Martinsson, and V. Rokhlin, Fast direct solvers for integral equations in complex three-dimensional domains, Acta Numerica, 18 (2009), 243275.

[28] L. Greengard and M. C. Kropinski, Integral equation methods for Stokes flow in doublyperiodic domains, J. Engrg. Math., 48 (2004), 157-170.

[29] L. Greengard, M. C. Kropinski, and A. Mayo, Integral equation methods for Stokes flow and isotropic elasticity in the plane, J. Comput. Phys., 125 (1996), 403-414.

[30] L. Greengard and J.Y. Lee, Accelerating the nonuniform fast Fourier transform, SIAM Rev., 46 (2004), 443-454.

[31] L. Greengard and P. Lin, Spectral approximation of the free-space heat kernel, Appl. Comput. Harmon. Anal., 9 (2000), 83-97.

[32] L. Greengard and V. Rokhlin, A Fast Algorithm for Particle Simulations, J. Comp. Phys., 73 (1987), 325-348. 
[33] L. Greengard and V. Rokhlin, A New Version of the Fast Multipole Method for the Laplace Equation in Three Dimensions, Acta Numerica, 6 (1997), 229-269.

[34] L. Greengard and J. Strain, The fast Gauss transform, SIAM J. Sci. Statist. Comput., 12 (1991), 79-94.

[35] L. Greengard and J. Strain, A fast algorithm for the evaluation of heat potentials, Comm. Pure Appl. Math., 43 (1990), 949-963.

[36] L. Greengard and X. Sun, A new version of the fast Gauss transform, Documenta Mathematica III (1998), 575-584.

[37] R. B. Guenther and J. W. Lee, Partial Differential Equations of Mathematical Physics and Integral Equations, Dover, 1988.

[38] R. B. Guenther and E. A. Thomann, Fundamental Solutions of Stokes and Oseen Problem in Two Spatial Dimensions, J. Math. Fluid Mech., 9 (2007), 489-505.

[39] W. Hackbusch (1999), A sparse matrix arithmetic based on H-matrices. Part I: In- troduction to H-matrices, Computing, 62, 89-108.

[40] W. Hackbusch and B. N. KhoromskiJ, A sparse $\mathcal{H}$-matrix arithmetic. Part II: Application to multi-dimensional problems, Computing, 64 (2000), 21-47.

[41] M. Hasegawa, M. Onishi, And M. Soya, Fundamental solution for transient incompressible viscous flow and its application to the two dimensional problem, Structual Eng./ Earthquake Eng., 3 (1986), 23-32.

[42] K.L. Ho AND L.GreEnGARD, A fast direct solver for structured linears systems by recursive skeletonization, arXiv:1110.3105.

[43] S. Jiang, Potential theory for initial-boundary value problems of unsteady Stokes flow in two dimensions, CAMS report 1112-8, New Jersey Institute of Technology, Newark, New Jersey, 2011.

[44] S. Kapur AND D. Long, IES $S^{3}$ : a fast integral equation solver for efficient 3-D extraction, in Proc. IEEE International Conference on Computer Aided Design, November 1997, 448-455.

[45] S. KAPUR AND V. RokHLIN, High-order corrected trapezoidal quadrature rules for singular functions, SIAM J. Numer. Anal., 34 (1997), 1331-1356.

[46] O. Kellog, Foundations of potential theory, Dover, New York, 1954.

[47] S. Kim And S. J. Karrila, Microhydrodynamics: Principles and Selected Applications, Dover, New York, 2005.

[48] R. Kress, Linear Integral Equations, Second Edition, Springer, 1999.

[49] O. A. Ladyzhenskaya. The mathematical theory of viscous imcompressible flow, Gordon \& Breach, 1969.

[50] J.Y. Lee and L. Greengard, The type 3 nonuniform FFT and its applications, J. Comput. Phys., 206 (2005), 1-5.

[51] J. E. LEwIS, The Initial-Boundary Value Problem for the Navier-Stokes Equations with Data in $L^{p}$, Indiana Univ. Math. J., 22 (1972/73), 739-761.

[52] J. Li AND L. Greengard, On the numerical solution of the heat equation. I. Fast solvers in free space, J. Comput. Phys., 226 (2007), 1891-1901.

[53] J. Li And L. GReEnGaRd, High order accurate methods for the evaluation of layer heat potentials, SIAM J. Sci. Comput., 31 (2009), 3847-3860.

[54] Y. Liu, Fast Multipole Boundary Element Method: Theory and Applications in Engineering, Cambridge University Press, New York, NY, USA, 2009.

[55] J. Ma, V. Rokhlin, And S. Wandzura, Generalized Gaussian quadrature rules for systems of arbitrary functions, SIAM J. Numer. Anal., 33 (1996), 971-996.

[56] P.G. Martinsson, A fast direct solver for a class of elliptic partial differential equations, SIAM J. Sci. Comput., 38 (2009), 316-330.

[57] P.G. Martinsson And V. Rokhlin, A fast direct solver for boundary integral equations in two dimensions, J. Comput. Phys., 205 (2005), 1-23.

[58] P. G. Martinsson and V. Rokhlin, An accelerated kernel-independent fast multipole method in one dimension, SIAM J. Sci. Comput., 29 (2007), 1160-1178.

[59] E. A. McIntyre, Boundary Integral Solutions of the Heat Equation, Math Comp., 46 (1986), $71-79$.

[60] E. Michielssen, A. Ergin, B. Shanker, and D. Weile, The multilevel plane wave time domain algorithm and its applications to the rapid solution of electromagnetic scattering problems: a review, Mathematical and numerical aspects of wave propagation (Santiago de Compostela, 2000), 24-33, SIAM, Philadelphia, PA.

[61] S. G. Mikhlin and S. Prossdorf, Singular integral operators, Springer-Verlag, Berlin, 1986.

[62] N. I. Muskhelishvili, Singular integral equations, Noordhoff, Groningen, 1953.

[63] N. Nishimura, Fast multipole accelerated boundary integral equation methods, Appl. Mech. Rev., 55 (2002), 299-324. 
[64] F. Odqvist, Beirtäge zur Theorie der nichtstationären zähen Flüssigkeiten, Arkiv för Matematik, Astronomie och Fysik, Bd 22 A, Hf 4 (1932), 1-22.

[65] C. W. Oseen, Neuere Methoden und Ergebnisse in der Hydrodynamik, Leipzig, Akademische Verlagsgesellschaft, 1927.

[66] J. R. Phillips And J. White, A precorrected-FFT method for electrostatic analysis of complicated 3-D structures, IEEE Trans. Comput. Aid. Design, 16 (1997), 1059-1072.

[67] W. Pogorzelski, Integral equations and their applications, Pergamon Press, 1966.

[68] D. Potts, G. Steidl, And M. TAsche, Fast Fourier transforms for nonequispaced data: A tutorial, in: J.J. Benedetto, P. Ferreira (Eds.), Modern Sampling Theory Mathematics and Applications, Birkhauser, Boston, 2001, 249-274.

[69] C. PozRikidis, Boundary integral and singularity methods for linearized viscous flow, Cambridge, 1992.

[70] Y. SAAD AND M. H. SChUltz, GMRES: a generalized minimum residual algorithm for solving nonsymmetric linear systems, SIAM J. Sci. Stat. Comput., 7 (1986), 856-869.

[71] Z. Shen, Boundary Value Problems for Parabolic Lamé Systems and a Nonstationary Linearized System of Navier-Stokes Equations in Lipschitz Cylinders, Am. J. of Math., 113 (1991), 293-373.

[72] V. A. SolonNikov, Estimates for solutions of a non-stationary linearized system of NavierStokes equations, Trudy Mat. Inst. Steklov., 70 (1964), 213-317.

[73] M. Spivak, S. K. Veerapaneni, and L. Greengard, The fast generalized Gauss transform, SIAM J. Sci. Comput., 32 (2010), 3092-3107.

[74] I. Stakgold, Boundary value problems of mathematical physics, Vol. I and II, Macmillan, 1968.

[75] E. M. Stein, Singular Integrals and differentiability properties of functions, Princeton University Press, 1970.

[76] E. M. Stein and G. Weiss, Introduction to Fourier analysis on Euclidean spaces, Princeton University Press, 1971.

[77] J. Strain, The fast Gauss transform with variable scales, SIAM J. Sci. Stat. Comput., 12 (1991), 1131-1139.

[78] J. Strain, Fast adaptive methods for the Free-space heat equation, SIAM J. Sci. Comput., 15 (1994), 185-206.

[79] T. Takahashi, N. Nishimura, and S. Kobayashi, A fast BIEM for three-dimensional elastodynamics in time domain, Engineering Analysis with Boundary Elements, 27 (2003), 491-506.

[80] C. C. Tsai, D. L. Young, C. M. Fan, And C. W. Chen, MFS with time-dependent fundamental solutions for unsteady Stokes equations, Engng. Anal. Boundary Elements, 30 (2006), 897908.

[81] S. K. Veerapaneni And G. Biros, A high-order solver for the heat equation in $1 D$ domains with moving boundaries, SIAM J. Sci. Comput., 29 (2007), 2581-2606.

[82] S. K. Veerapaneni and G. Biros, The Chebyshev fast Gauss and nonuniform fast Fourier transforms and their application to the evaluation of distributed heat potentials, J. Comput. Phys., 227 (2008), 7768-7790.

[83] N. YARVIN AND V. Rokhlin, Generalized Gaussian quadratures and singular value decompositions of integral operators, SIAM J. Sci. Comput., 20 (1998), 699-718.

[84] L. YING, A kernel independent fast multipole algorithm for radial basis functions, J. of Comput. Phys., 213 (2006), 451-457.

[85] L. YING, G. BIROS, AND D. ZORIN, A kernel-independent adaptive fast multipole algorithm in two and three dimensions, J. Comput. Phys., 196 (2004), 591-626.

[86] L. Ying, G. Biros, AND D. Zorin, A high-order 3D boundary integral equation solver for elliptic PDEs in smooth domains, J. Comput. Phys., 219 (2006), 247-275.

[87] K. Yoshida, N. Nishimura, And S. Kobayashi, Application of New Fast Multipole Boundary Integral Equation Method to Crack Problems in 3D, Eng. Anal. Boundary Elements, 25 (2001), 239-247.

[88] B. ZhANG, Integral-Equation-Based Fast Algorithms and Graph-Theoretic Methods for LargeScale Simulations. PhD thesis, University of North Carolina, Chapel Hill, 2010. 